\title{
CAPTURA, CUANTIFICACIÓN Y CARACTERIZACIÓN DEL MATERIAL PARTICULADO SEDIMENTABLE EN TECHUMBRES DE LA CIUDAD DE COPIAPÓ
}

\author{
CAPTURE, QUANTIFICATION AND CHARACTERIZATION OF THE \\ SETTLEABLE PARTICULATED MATERIAL ON COPIAPÓ CITY ROOFS
}

\author{
Leonardo Villarroel S. ${ }^{1}$; José R. Morales ${ }^{2}$; Pedro Miranda J. ${ }^{2}$; \\ Claudio Díaz S. ${ }^{1}$; Nils Arce M. ${ }^{1}$; Claudio Campos ${ }^{1}$
}

\begin{abstract}
RESUMEN
Se realizó la caracterización del material particulado depositado por las empresas mineras de la ciudad de Copiapó en los relaves de mayor impacto ambiental, así como la concentración de algunos de los elementos presentes en ellos. Simultáneamente, se procedió a capturar, cuantificar y caracterizar el material particulado suspendido y precipitable presente en la columna de aire disponible para la población de la ciudad de Copiapó y determinar, además, el porcentaje de los elementos detectados en cada muestra. La caracterización del material particulado, tanto de los relaves como el capturado en la columna de aire y los recolectores PM10 de alto volumen, fue realizada mediante los análisis PIXE (Proton Induced X-ray Emission) y GIXE (Gamma Induced $\mathrm{X}$-ray Emission). Estos análisis no detectaron la presencia de los elementos más dañinos para la salud humana, a saber, cadmio y arsénico, al menos en puntos cercanos a la ciudad de Copiapó; sin embargo, se logró determinar la presencia de plomo en una zona de alta densidad poblacional.
\end{abstract}

Palabras clave: Contaminación del aire, material particulado.

\begin{abstract}
The characterization of the particulated material from highly environmental impact tailings settled by mining companies of Copiapó was carried out together with the concentration of some of the elements they contain. Simultaneously, the suspended settleable particulated material that contains the air column available for the population in Copiapó was captured, quantified and characterized and the percentage of the elements present in each sample was determined. The characterization of the particulated material both from the tailings and from the air column and the PM10 high volume collectors, was carried out by PIXE (Proton Induced X-ray Emission) and GIXE (Gamma Induced X-ray Emission) analysis. These analyses did not detect the presence of harmful elements for human health, such as cadmium and arsenic, at least in nearby points to the city. Though it was possible to detect the presence of lead in a highly populated area.

Key words: Pollution of the air, particulated material.
\end{abstract}

\section{INTRODUCCIÓN}

Copiapó es una ciudad estrechamente ligada a la actividad minera, sin embargo no existe la información deseable sobre el material particulado en suspensión en las vecindades de las poblaciones y que eventualmente podría estar ingresando al organismo de sus habitantes por vía aérea o digestiva. Las fuentes contaminantes son múltiples, dado que en su entorno existen muchos relaves mineros abandonados y otros en pleno crecimiento debido a plantas procesadoras activas, además de

1 Universidad de Atacama, Facultad de Ciencias Naturales, Departamento de Física. Casilla 240. Copiapó, Chile. leonardo. villarroell@uda.cl; claudio.diaz@uda.cl

2 Universidad de Chile, Facultad de Ciencias, Departamento de Física. Casilla 653. Santiago, Chile.rmorales@uchile.cl

Fecha de Recepción:15 Diciembre 2008

Fecha de Aceptación: 23 Marzo 2009 
algunas fundiciones. En particular, es de interés, por el riesgo que significa para la salud de la población, estudiar la posible existencia a nivel de traza de elementos tóxicos como $\mathrm{Cu}, \mathrm{Fe}$, As, $\mathrm{Pb}$, entre otros.

\section{METODOLOGÍA}

\section{RELAVES}

Se tomaron muestras de cinco relaves localizados en Copiapó, a saber, Hochschild Norte, Palomar 1, Palomar 2, San Esteban y Hochschild Uda, ubicados aproximadamente a $7[\mathrm{~km}], 3[\mathrm{~km}]$, $3[\mathrm{~km}], 5[\mathrm{~km}], 1,5[\mathrm{~km}]$ del centro de Copiapó, res- pectivamente. De cada relave se tomaron muestras de cinco puntos diferentes, separados entre $30 \mathrm{y}$ $100 \mathrm{~m}$ entre ellos. El material recolectado fue depositado en un recipiente común y mediante agitación se obtuvo una mezcla homogénea y representativa. De cada una de las mezclas ya homogeneizadas se prepara la muestra definitiva tomando material de cinco puntos distintos de la mezcla homogeneizada, las cuales fueron ingresadas a sendos portamuestras, procediendo a homogeneizarlas mediante agitación. Finalmente estos cinco portamuestras fueron enviados al Centro de Estudios de Física Experimental de la Universidad de Chile (CEFEX).

La siguiente figura muestra los puntos donde se recolectaron las muestras de relaves:

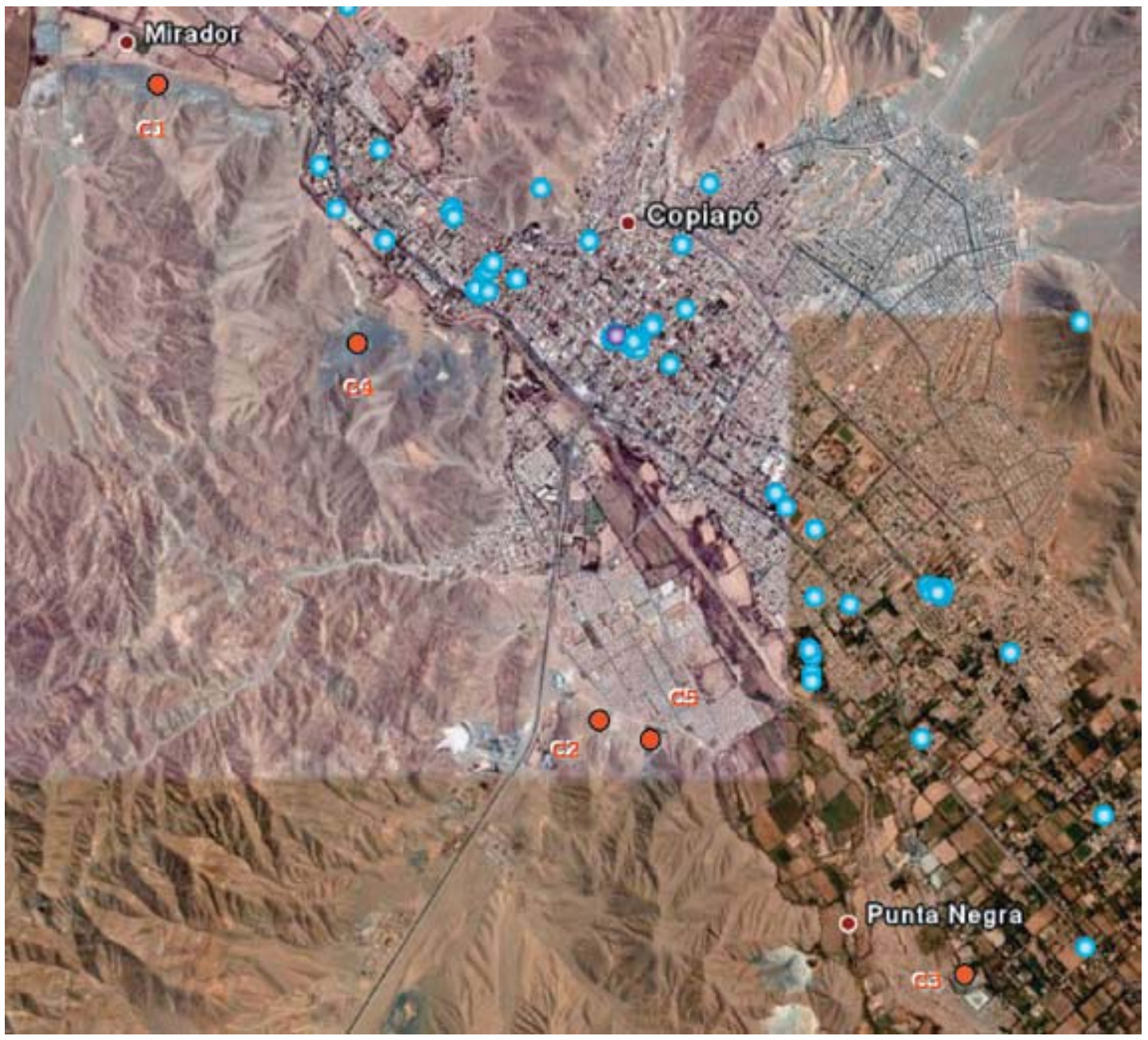

Figura 1. Ubicación de los relaves en la ciudad de Copiapó (•). 
MATERIAL PARTICULADO RECOLECTADO A NIVEL DE TECHUMBRES

Se colocaron trampas para capturar material particulado en suspensión a nivel de las techumbres en cinco conjuntos habitacionales situados, en promedio, a $3[\mathrm{~km}]$ del centro de Copiapó durante los meses de agosto y septiembre, recolectando en promedio $0,892 \mathrm{~g}$ con una dispersión de $0,44 \mathrm{~g}$.

El particulado, recolectado en las trampas, se homogeneizó mediante agitación y los portamuestras respectivos fueron enviados al CEFEX para su análisis PIXE (Proton Induced X-ray Emisión) y GIXE (Gamma Induced X-ray Emisión).

\section{RECOLECTORES PMIO DE ALTO VOLUMEN}

Complementariamente, se instalaron recolectores PM10 de alto volumen en dos zonas representati- vas de Copiapó, los cuales capturaron el material particulado de diámetro menor a $10[\mu \mathrm{m}]$ durante 48 y 72 horas, respectivamente. Los filtros respectivos fueron cambiados cada 24 horas. Dichos filtros fueron enviados al CEFEX para sus análisis respectivos.

\section{RESULTADOS Y DISCUSIÓN}

\section{ANÁLISIS GIXE}

\section{Relaves}

Con las muestras de relave se confeccionaron pastillas (pellets) que fueron analizadas por el método GIXE usando una fuente radiactiva de Am-241.

Los resultados se muestran en página siguiente:

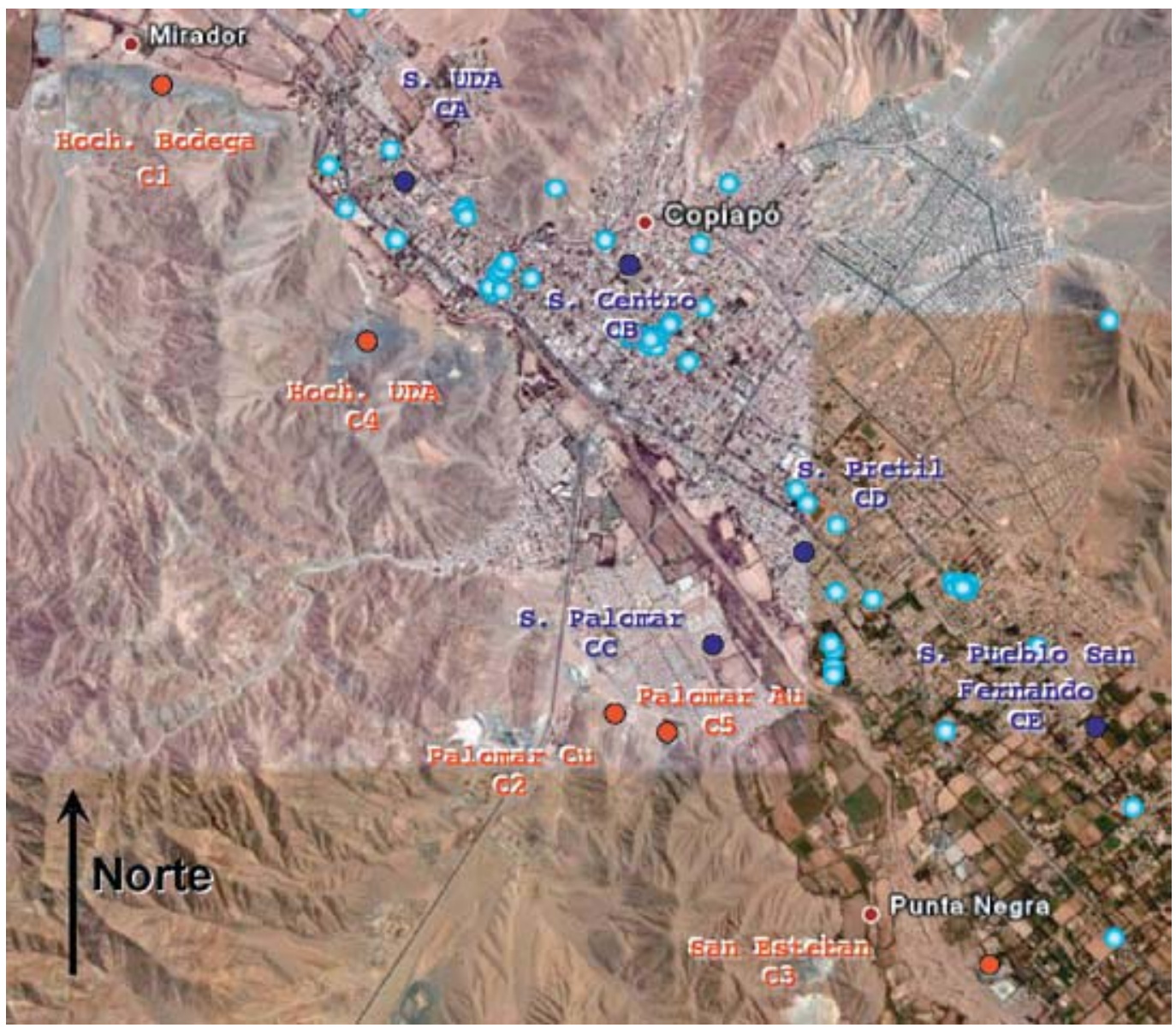

Figura 2. Ubicación de las trampas recolectoras en la ciudad de Copiapó (•). 
Tabla 1

Concentración de Fe y Ba de relaves C1, C4 y C5

\begin{tabular}{|l|c|c|c|}
\hline Muestra & $\mathbf{F e}$ & $\mathbf{B a}$ & $\mathbf{F e} /(\mathbf{B a} * \mathbf{1 0 0})$ \\
\hline & $\%$ & $0.01 \%$ & \\
\hline C 1 & 2.40 & 4.05 & 0.59 \\
\hline C 4 & 2.83 & 2.52 & 1.12 \\
\hline C 5 & 3.49 & 7.04 & 0.50 \\
\hline Promedio & 2.91 & 4.54 & 0.74 \\
\hline
\end{tabular}

La concentración para el $\mathrm{Fe}$ está en tanto por ciento y la del bario en centésimas de porciento. Se ha calculado el cuociente entre ambas concentraciones.

La muestra $\mathrm{C} 5$ tiene los mayores valores en ambos elementos. El cuociente ( $\mathrm{Fe} / \mathrm{Ba}$ ) es semejante en las muestras $\mathrm{C} 1$ y $\mathrm{C} 5$.

$\mathrm{La}$ abundancia relativa de $\mathrm{Fe}$ con respecto a Ba es mayor en la muestra $\mathrm{C} 4$, lo que podría ser una característica del relave en que se recogió el material.

Las mismas pastillas se irradiaron con la fuente radiactiva de Cd-109. Cabe hacer notar que esta fuente no permite detectar el elemento $\mathrm{Ba}$.

Tabla 2

Concentración de Fe de relaves C1, C4 y C5

\begin{tabular}{|l|c|}
\hline \multicolumn{1}{|c|}{ Muestra } & Fe\% \\
\hline C 1 & 2.16 \\
\hline C 4 & 2.89 \\
\hline C 5 & 3.43 \\
\hline Promedio & 2.83 \\
\hline
\end{tabular}

Los resultados obtenidos para el Fe con ambas fuentes son semejantes.

\section{PARTICULADO RECOLECTADO A NIVEL DE} TECHUMBRES

Los pellets fabricados con las muestras CA, $\mathrm{CB}, \mathrm{CC}, \mathrm{CD}$ y $\mathrm{CE}$ se irradiaron con las fuentes de
Am-241 y Cd-109. Los resultados se muestran a continuación:

Tabla 3

Concentración de Fe y Ba a nivel de techumbres

\begin{tabular}{|l|c|c|c|}
\hline & Am-241 & Cd-109 & Am-241 \\
\hline Muestra & Fe\% & Fe\% & Ba 0.01\% \\
\hline CA & 2.14 & 1.07 & 6.18 \\
\hline CB & 1.74 & 1.09 & 6.07 \\
\hline CC & 2.03 & 1.15 & 7.67 \\
\hline CD & 1.78 & 1.08 & 6.80 \\
\hline CE & 1.63 & 0.98 & 5.42 \\
\hline & 1.86 & 1.07 & 6.43 \\
\hline
\end{tabular}

Se puede observar que, en general, las concentraciones de Fe determinadas por ambas fuentes son menores que las mostradas en las tablas anteriores referente a las muestras 1,4 y 5 . En cambio, la concentración de $\mathrm{Ba}$ es algo mayor.

\section{ANÁLISIS PIXE}

\section{Relaves y particulado recolectado a nivel de techumbres}

Para peletizar las muestras se utilizó un aglomerante de polietileno. Con el objetivo de determinar las concentraciones absolutas, se utilizó un material de referencia NCS DC 78301 (River Sediment) que proviene del laboratorio CENMA de la Universidad de Chile, obteniéndose la siguiente tabla de concentraciones (Tabla 4).

Las concentraciones relativas al $\mathrm{Fe}$ se obtienen de la tabla anterior realizando el cuociente entre las concentraciones de los distintos compuestos dividido por la concentración del oxido de fierro $\left(\mathrm{Fe}_{2} \mathrm{O}_{3}\right)$. Las tablas de concentraciones relativas son las siguientes (Ver tablas 5 y 6).

El espectro obtenido para la muestra de relave $\mathrm{C} 1$ se muestra en el difractograma (Figura 3). 
Tabla 4

Concentraciones absolutas promedio de relaves $\mathrm{C} 1$ al $\mathrm{C} 5 \mathrm{y}$ material recolectado a nivel de techumbre.

\begin{tabular}{|l|l|c|c|c|c|c|c|c|c|c|c|c|c|c|}
\hline $\mathbf{Z}$ & Elemento & $\mathbf{K} \mathbf{a}$ & $\begin{array}{c}\text { Factor } \\
\text { Gravimétrico }\end{array}$ & $\mathbf{C 1}$ & $\mathbf{C 2}$ & $\mathbf{C 3}$ & $\mathbf{C 4}$ & $\mathbf{C 5}$ & $\mathbf{C A}$ & $\mathbf{C B}$ & $\mathbf{C C}$ & $\mathbf{C D}$ & $\mathbf{C E}$ & CENMA \\
\hline 12 & $\mathrm{MgO}$ & 1,04 & 0,6030 & 0,5 & 0,6 & 1,9 & 0,6 & 1,1 & 0,8 & 0,6 & 0,6 & 0,7 & 0,6 & 0,5 \\
\hline 13 & $\mathrm{~A} 2 \mathrm{O} 3$ & 1,49 & 0,5293 & 5,7 & 5,1 & 8,6 & 4,5 & 8,1 & 9,7 & 9,7 & 7,5 & 8,8 & 9,0 & 12,4 \\
\hline 14 & $\mathrm{SiO} 2$ & 1,74 & 0,4674 & 25,1 & 17,2 & 33,7 & 22,0 & 33,1 & 31,5 & 37,0 & 28,6 & 30,7 & 34,4 & 60,4 \\
\hline 15 & $\mathrm{P} 2 \mathrm{O} 5$ & 2,02 & 0,4364 & 0,15 & 0,08 & 0,15 & 0,11 & 0,16 & 0,15 & 0,22 & 0,12 & 0,14 & 0,08 & 0,17 \\
\hline 16 & $\mathrm{~S}$ & 2,31 & 1,0000 & 0,14 & 0,04 & 0,11 & 0,21 & 0,17 & 0,27 & 0,27 & 0,22 & 0,27 & 0,23 & 0,00 \\
\hline 17 & $\mathrm{Cl}$ & 2,62 & 1,0000 & 0,01 & 0,01 & 0,02 & 0,01 & 0,01 & 0,19 & 0,23 & 0,14 & 0,16 & 0,16 & 0,00 \\
\hline 19 & $\mathrm{~K} 2 \mathrm{O}$ & 3,31 & 0,8301 & 0,39 & 0,32 & 0,63 & 0,11 & 0,69 & 0,90 & 1,22 & 0,78 & 0,91 & 1,15 & 2,03 \\
\hline 20 & $\mathrm{CaO}$ & 3,69 & 0,7147 & 4,3 & 0,5 & 1,4 & 4,4 & 1,0 & 4,4 & 4,1 & 3,8 & 4,5 & 4,8 & 0,31 \\
\hline 22 & $\mathrm{TiO} 2$ & 4,51 & 1,1987 & 0,08 & 0,10 & 0,13 & 0,05 & 0,09 & 0,22 & 0,22 & 0,15 & 0,15 & 0,20 & 0,42 \\
\hline 24 & $\mathrm{Cr} 2 \mathrm{O} 3$ & 5,41 & 0,6827 & 0,02 & 0,02 & 0,02 & 0,02 & 0,01 & 0,03 & 0,02 & 0,01 & 0,02 & 0,03 & 0,02 \\
\hline 25 & $\mathrm{MnO}$ & 5,90 & 0,7745 & 0,08 & 0,00 & 0,05 & 0,04 & 0,02 & 0,05 & 0,06 & 0,08 & 0,06 & 0,06 & 0,09 \\
\hline 26 & $\mathrm{FeO}$ & 6,40 & 0,7773 & 1,3 & 1,3 & 2,3 & 1,4 & 2,2 & 0,6 & 0,6 & 0,4 & 0,4 & 0,4 & 0,68 \\
\hline 26 & $\mathrm{Fe} 2 \mathrm{O} 3$ & 6,40 & 0,6994 & 8,8 & 8,6 & 15,4 & 9,3 & 14,7 & 4,0 & 3,7 & 2,9 & 2,9 & 2,9 & 4,5 \\
\hline 29 & $\mathrm{Cu} 2 \mathrm{O}$ & 8,05 & 0,8878 & 0,09 & 0,03 & 0,21 & 0,20 & 0,16 & 0,11 & 0,11 & 0,10 & 0,14 & 0,10 & 0,05 \\
\hline 30 & $\mathrm{ZnO}$ & 8,64 & 0,8034 & 0,01 & 0,01 & 0,04 & 0,02 & 0,03 & 0,05 & 0,07 & 0,04 & 0,02 & 0,03 & 0,05 \\
\hline 82 & $\mathrm{~Pb}$ & 14,77 & 1,0000 & 0,06 & 0,04 & 0,08 & 0,08 & 0,08 & 0,04 & 0,02 & 0,05 & 0,13 & 0,03 & 0,05 \\
\hline
\end{tabular}

Tabla 5

Concentraciones relativas a Fe de relaves $\mathrm{C} 1$ al $\mathrm{C5}$.

\begin{tabular}{|c|l|r|r|r|r|r|r|r|}
\hline $\begin{array}{c}\text { Concentraciones } \\
\text { Relativas a Fe }\end{array}$ & & & \multicolumn{1}{|c|}{$\mathbf{C 1}$} & \multicolumn{1}{c|}{$\mathbf{C 2}$} & \multicolumn{1}{c|}{$\mathbf{C 3}$} & \multicolumn{1}{c|}{$\mathbf{C 4}$} & C5 & CENMA \\
\hline $\mathbf{Z}$ & Elemento & $\mathbf{K} \_\mathbf{a}$ & & & & & & \\
\hline 12 & $\mathrm{MgO}$ & 1,04 & 6,268 & 7,037 & 12,587 & 6,272 & 20,109 & 11,953 \\
\hline 13 & $\mathrm{Al2O} 3$ & 1,49 & 64,959 & 59,651 & 55,675 & 48,179 & 52,003 & 277,014 \\
\hline 14 & $\mathrm{SiO} 2$ & 1,74 & 286,315 & 199,771 & 218,253 & 236,926 & 159,309 & 1352,807 \\
\hline 15 & $\mathrm{P} 2 \mathrm{O} 5$ & 2,02 & 1,669 & 0,937 & 0,966 & 1,182 & 2,078 & 3,704 \\
\hline 16 & $\mathrm{~S}$ & 2,31 & 1,557 & 0,432 & 0,692 & 2,222 & 2,735 & 0,066 \\
\hline 17 & $\mathrm{Cl}$ & 2,62 & 0,140 & 0,169 & 0,120 & 0,128 & 0,195 & 0,079 \\
\hline 19 & $\mathrm{~K} 2 \mathrm{O}$ & 3,31 & 4,404 & 3,733 & 4,105 & 1,227 & 0,841 & 45,553 \\
\hline 20 & $\mathrm{CaO}$ & 3,69 & 49,490 & 6,228 & 9,236 & 47,644 & 61,022 & 7,041 \\
\hline 22 & $\mathrm{TiO} 2$ & 4,51 & 0,950 & 1,172 & 0,814 & 0,532 & 1,451 & 9,438 \\
\hline 24 & $\mathrm{Cr} 2 \mathrm{O} 3$ & 5,41 & 0,284 & 0,222 & 0,102 & 0,215 & 0,514 & 0,384 \\
\hline 25 & $\mathrm{MnO}$ & 5,90 & 0,964 & 0,039 & 0,352 & 0,432 & 0,136 & 2,127 \\
\hline 26 & $\mathrm{FeO}$ & 6,40 & 15,210 & 15,210 & 15,210 & 15,210 & 15,210 & 15,210 \\
\hline 26 & $\mathrm{Fe} 2 \mathrm{O} 3$ & 6,40 & 100,000 & 100,000 & 100,000 & 100,000 & 100,000 & 100,000 \\
\hline 29 & $\mathrm{Cu} 2 \mathrm{O}$ & 8,05 & 1,051 & 0,306 & 1,375 & 2,142 & 0,364 & 1,071 \\
\hline 30 & $\mathrm{ZnO}$ & 8,64 & 0,105 & 0,109 & 0,276 & 0,208 & 3,198 & 1,055 \\
\hline 82 & $\mathrm{~Pb}$ & 14,77 & 0,630 & 0,507 & 0,496 & 0,893 & 2,878 & 1,071 \\
\hline
\end{tabular}


Tabla 6

Concentraciones relativas a Fe para particulado recolectado a nivel de techumbre

\begin{tabular}{|c|l|r|r|r|r|r|r|r|}
\hline $\begin{array}{c}\text { Concentraciones } \\
\text { Relativas a Fe }\end{array}$ & & & CA & \multicolumn{1}{c|}{ CB } & CC & CD & CE & CENMA \\
\hline $\mathbf{Z}$ & Elemento & K_a & & & & & & \\
\hline 12 & $\mathrm{MgO}$ & 1,04 & 18,774 & 15,611 & 21,864 & 23,562 & 20,015 & 11,953 \\
\hline 13 & $\mathrm{Al2O} 3$ & 1,49 & 242,984 & 262,872 & 253,224 & 306,650 & 308,079 & 277,014 \\
\hline 14 & $\mathrm{SiO} 2$ & 1,74 & 785,581 & 998,555 & 970,470 & 1072,551 & 1171,968 & 1352,807 \\
\hline 15 & $\mathrm{P} 2 \mathrm{O} 5$ & 2,02 & 3,670 & 5,933 & 4,234 & 4,800 & 2,825 & 3,704 \\
\hline 16 & $\mathrm{~S}$ & 2,31 & 6,680 & 7,322 & 7,423 & 9,538 & 7,779 & 0,066 \\
\hline 17 & $\mathrm{Cl}$ & 2,62 & 4,630 & 6,305 & 4,803 & 5,591 & 5,599 & 0,079 \\
\hline 19 & $\mathrm{~K} 2 \mathrm{O}$ & 3,31 & 22,522 & 32,848 & 26,421 & 31,577 & 39,150 & 45,553 \\
\hline 20 & $\mathrm{CaO}$ & 3,69 & 109,000 & 110,282 & 129,708 & 155,794 & 165,201 & 7,041 \\
\hline 22 & $\mathrm{TiO} 2$ & 4,51 & 5,440 & 5,993 & 4,954 & 5,277 & 6,900 & 9,438 \\
\hline 24 & $\mathrm{Cr} 2 \mathrm{O} 3$ & 5,41 & 0,748 & 0,597 & 0,505 & 0,717 & 0,926 & 0,384 \\
\hline 25 & $\mathrm{MnO}$ & 5,90 & 1,359 & 1,508 & 2,834 & 2,065 & 1,952 & 2,127 \\
\hline 26 & $\mathrm{FeO}$ & 6,40 & 15,210 & 15,210 & 15,210 & 15,210 & 15,210 & 15,210 \\
\hline 26 & $\mathrm{Fe} 2 \mathrm{O} 3$ & 6,40 & 100,000 & 100,000 & 100,000 & 100,000 & 100,000 & 100,000 \\
\hline 29 & $\mathrm{Cu} 2 \mathrm{O}$ & 8,05 & 2,707 & 3,067 & 3,271 & 4,913 & 3,338 & 1,071 \\
\hline 30 & $\mathrm{ZnO}$ & 8,64 & 1,306 & 1,773 & 1,202 & 0,670 & 1,032 & 1,055 \\
\hline 82 & $\mathrm{~Pb}$ & 14,77 & 0,915 & 0,518 & 1,564 & 4,482 & 0,894 & 1,071 \\
\hline
\end{tabular}

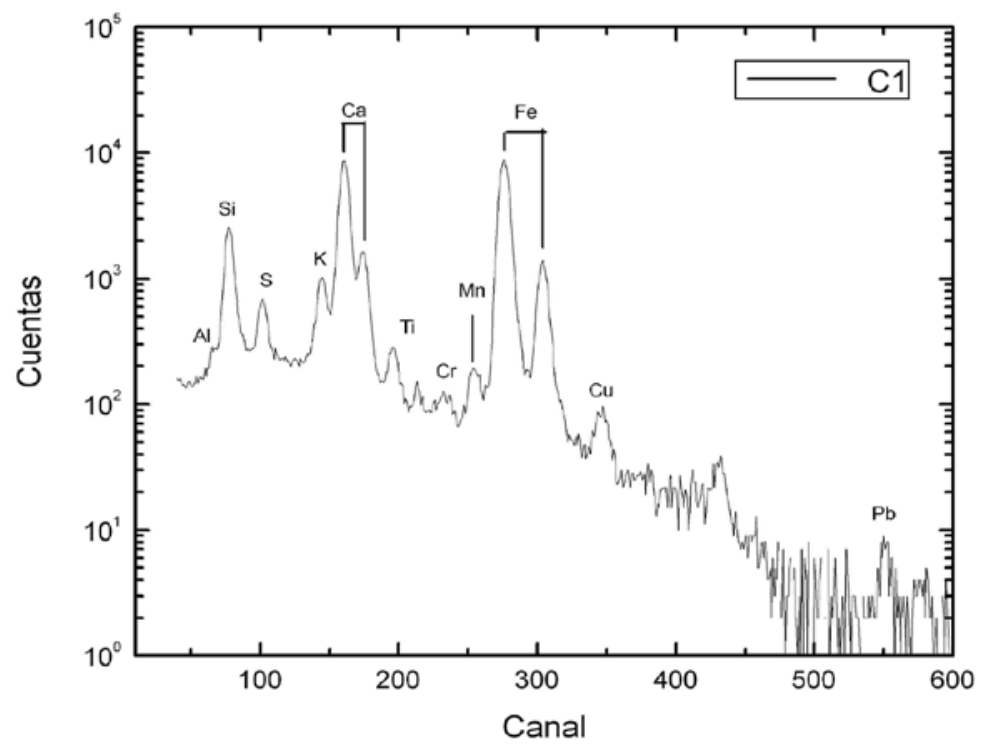

Figura 3. Difractograma relave C1- Hochschild Bodega. 
Análogamente, el difractograma correspondiente a la muestra de polvo recolectado a nivel de techumbre CA (Figura 4).

\section{RECOLECTORES PM10 DE ALTO VOLUMEN}

Los filtros de cuarzo cargados con material recolectado de los colectores de alto volumen PM10 se irradiaron con el método PIXE, encontrándose varios elementos, entre ellos $\mathrm{Al} \mathrm{y} \mathrm{Cu}$. Paralelamente se irradió un filtro virgen, y se encontró un espectro multielemental muy similar al anterior. Estas irradiaciones mostraron que el filtro de cuarzo enviado a análisis estaba contaminado, en altas cantidades, con elementos que participaron en su proceso de fabricación ( $\mathrm{Si}, \mathrm{Cl}, \mathrm{K}, \mathrm{Ca}, \mathrm{Fe}, \mathrm{Zn}, \mathrm{Ba}$ ), elementos que distorsionan el análisis del material depositado en él.

A fin de tener un estudio independiente del filtro virgen se realizó una cromatografía iónica a un trozo del filtro. El resultado fue concordante con el encontrado anteriormente en cuanto a que estos filtros se han contaminado o que los elementos presentes en ellos fueron incorporados en su proceso de fabricación. Para graficar esta situación, en la siguiente figura se muestra la comparación entre el filtro 933 y el filtro virgen (Figura 5).

No obstante lo anterior, este estudio permitió determinar la concentración del particulado PM10 en promedio de 24 horas en dos zonas seleccionadas de Copiapó (Tabla 7).

$\mathrm{Al}$ realizar una comparación con la tabla que la CONAMA Metropolitana utiliza para determinar la calidad del aire por partículas en la Región Metropolitana (Tabla 8) se puede observar que la calidad del aire para los días y lugares señalados se encuentra dentro del índice $0-10$, lo cual debe considerarse como bueno.

\section{ANÁLISIS DE LOS RESULTADOS}

Para un mejor análisis de este estudio se procede a hacer cuatro gráficos de barra para seis elementos, a saber, $\mathrm{P}_{2} \mathrm{O}_{5}, \mathrm{~S}, \mathrm{Cl}, \mathrm{Pb}, \mathrm{Al}_{2} \mathrm{O}_{3}$ y $\mathrm{Si}$. En esta selección se consideró, en parte, el impacto de estos elementos en la salud humana (Figura 6).

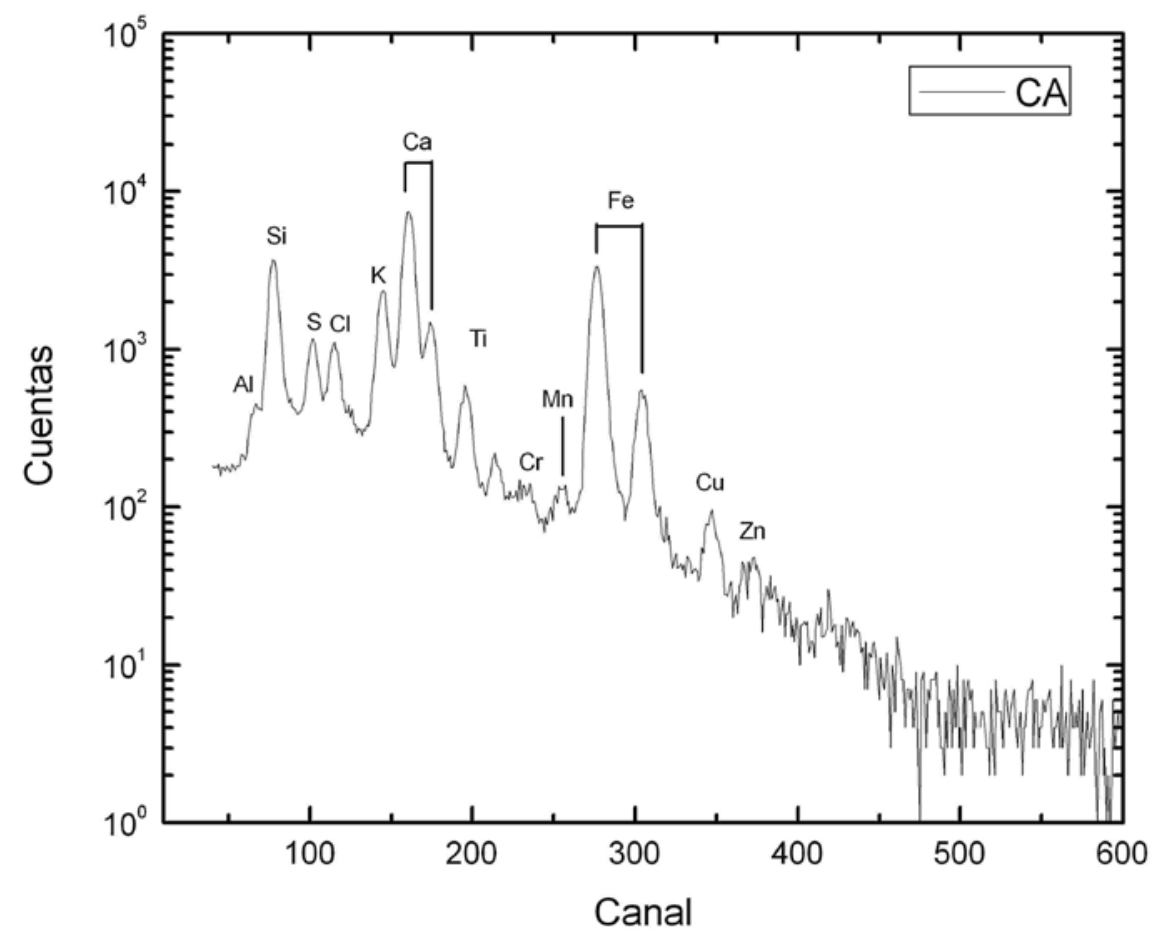

Figura 4. Difractograma CA - Sector UDA. 


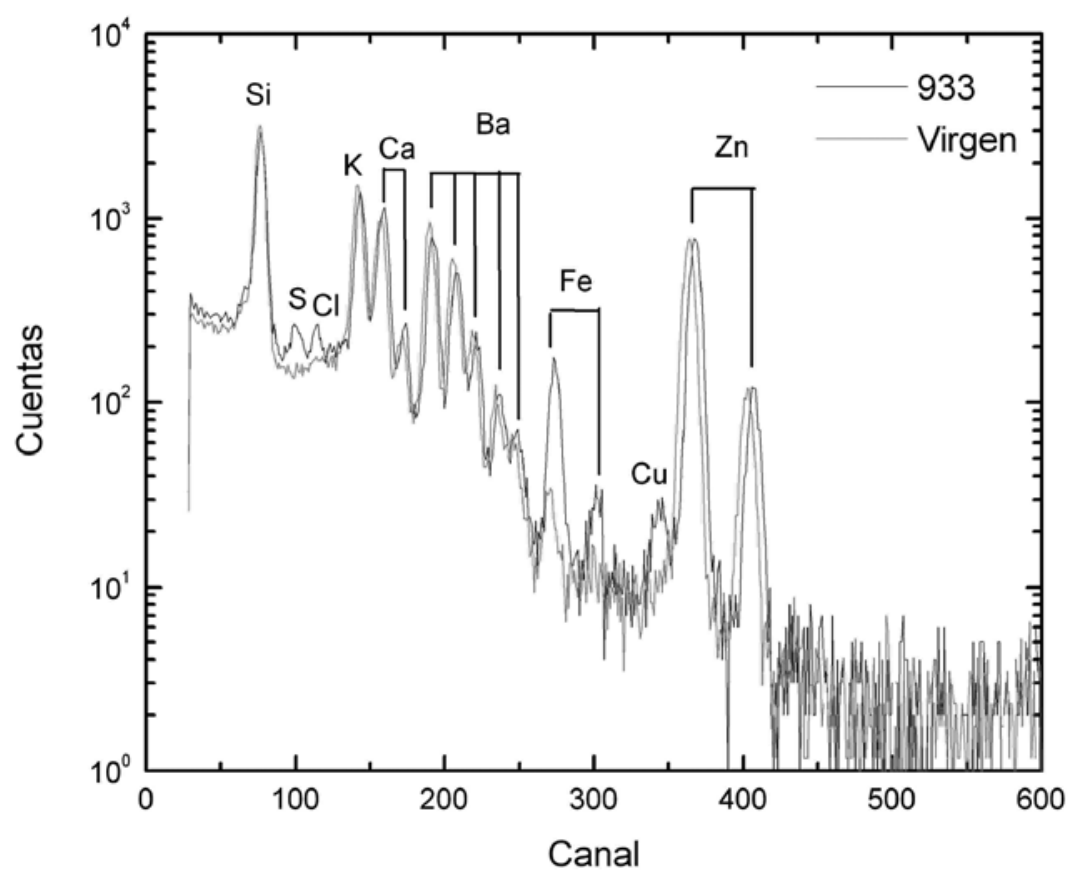

Figura 5. Difractograma filtro PM10 virgen v/s contaminado.

Tabla 7

Concentraciones particulado PM10.

\begin{tabular}{|c|c|c|c|c|c|}
\hline $\mathbf{N}^{\mathbf{0}}$ Filtro & Fecha & $\begin{array}{c}\text { Aire aspirado } \\
\left(\mathbf{2 4} \text { horas) } \mathbf{m}^{\mathbf{3}}\right.\end{array}$ & Masa Inicial & Masa Final & MP10 $\left(\boldsymbol{\mu g} / \mathbf{N m}^{\mathbf{3}}\right)$ \\
\hline 933 & 28-May & 1558 & 2,7877 & 2,9226 & $\mathbf{8 6 , 5}$ \\
\hline 934 & 29-May & 1563 & 2,805 & 2,947 & $\mathbf{9 0 , 8}$ \\
\hline 935 & 30-May & 1572 & 2,7793 & 2,932 & $\mathbf{9 7 , 1}$ \\
\hline 938 & 03-Jun & 1559 & 2,787 & 2,8812 & $\mathbf{6 0 , 4}$ \\
\hline 940 & 04-Jun & 1577 & 2,7905 & 2,8593 & $\mathbf{4 3 , 6}$ \\
\hline
\end{tabular}

Tabla 8

Indicadores de concentración CONAMA

\begin{tabular}{|l|c|c|}
\hline \multicolumn{1}{|c|}{ Estado } & Índice & Partículas \\
\hline Bueno & $0-10$ & $\left(0\right.$ a $\left.150 \mathrm{PM10} \mu \mathrm{g} / \mathrm{m}^{3}\right)$ \\
\hline Regular & $101-200$ & $\left(150\right.$ a $\left.195 \mathrm{PM} 10 \mu \mathrm{g} / \mathrm{m}^{3}\right)$ \\
\hline Alerta & $201-300$ & $\left(195\right.$ a $\left.239 \mathrm{PM} 10 \mu \mathrm{g} / \mathrm{m}^{3}\right)$ \\
\hline Preemergencia & $301-500$ & $\left(240\right.$ a $\left.329 \mathrm{PM} 10 \mu \mathrm{g} / \mathrm{m}^{3}\right)$ \\
\hline Emergencia & $501-$ más & $\left(330\right.$ o más PM10 $\left.\mu \mathrm{g} / \mathrm{m}^{3}\right)$ \\
\hline
\end{tabular}

Fuente: CONAMA. 
Porcentaje de elementos presentes

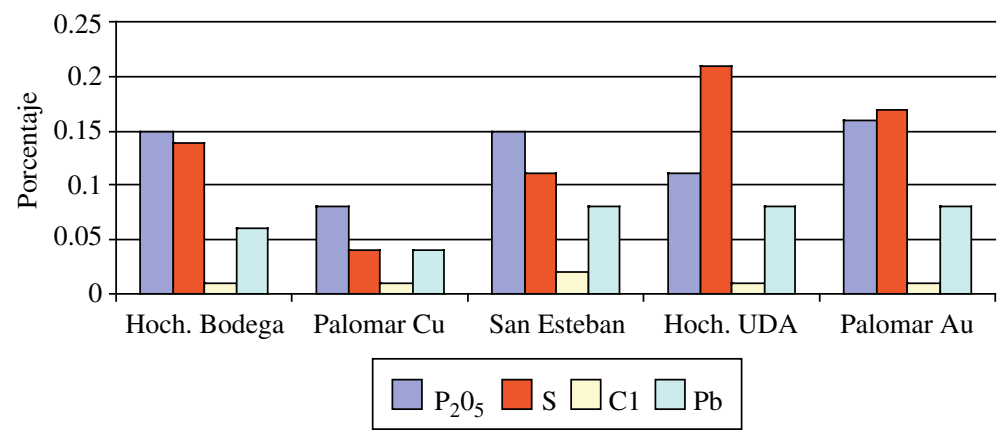

Figura 6. Porcentaje de concentración relaves.

Claramente se observa que el relave Palomar $\mathrm{Au}$ en comparación con el relave cercano Palomar $\mathrm{Cu}$ representa un mayor riesgo potencial para la salud de la población que existe en su entorno.

Con respecto a la concentración del compuesto $\mathrm{P}_{2} \mathrm{O}_{5}$, se observa que es prácticamente la misma en tres relaves y algo menor en los relaves Palomar $\mathrm{Cu}$ y Hoch. UDA, siendo este último el que presenta mayor concentración de azufre.

Como se puede apreciar en el siguiente gráfico las concentraciones de $\mathrm{Al}_{2} \mathrm{O}_{3}$ y $\mathrm{SiO}_{2}$ en el relave activo San Esteban son comparables a las mostradas en el relave pasivo Palomar Au. (Figura 7).

Los siguientes gráficos se refieren a la concentración obtenida de la recolección del particulado a nivel de techumbre en cinco poblaciones de la ciudad de Copiapó (Figura 8).

Se observa que la concentración de azufre es prácticamente uniforme en los cinco sectores, así como la concentración de cloro. En cambio, se nota una concentración de plomo mayoritaria en el sector El Pretil, siendo las restantes prácticamente uniformes.

Como se puede apreciar en el gráfico siguiente, a nivel poblacional, el particulado respirable logra cierta uniformidad para los componentes mostrados (Figura 9).

\section{CONCLUSIONES}

El análisis PIXE no permitió detectar, a nivel de trazas, los elementos cadmio y arsénico, lo cual nos indica que la concentración de estos elementos, de existir, es muy baja y menor a cinco partes por millón, pues este método no detecta concentraciones tan bajas. Esto es muy relevante dado que está comprobado que estos elementos son altamente dañinos para la salud humana.

En general, al observar la Tabla $\mathrm{N}^{\circ} 4$ se puede decir que no existe una uniformidad en la concentración del material particulado a nivel de techumbre en los distintos sectores poblacionales, notando que para algunos elementos la mayor concentración se presenta en el centro de la ciudad de Copiapó.

En este estudio no se puede sugerir una correlación explícita entre el material recolectado a nivel de techumbres y los relaves estudiados.

En la figura 9 se observa que en el sector El Pretil existe una concentración de plomo tres veces superior a la media de los otros cuatro sectores poblacionales. Lo anterior hace pensar que existe una fuente que emite plomo al ambiente en el entorno al Parque El Pretil, probablemente esto esté asociado a un proceso artesanal de recuperación del oro.

\section{AGRADECIMIENTO}

Los autores de este trabajo expresan su agradecimiento a la Dirección de Investigación y Postgrado de la Universidad de Atacama por el financiamiento otorgado al proyecto DIUDA $221155 / 2008$ 


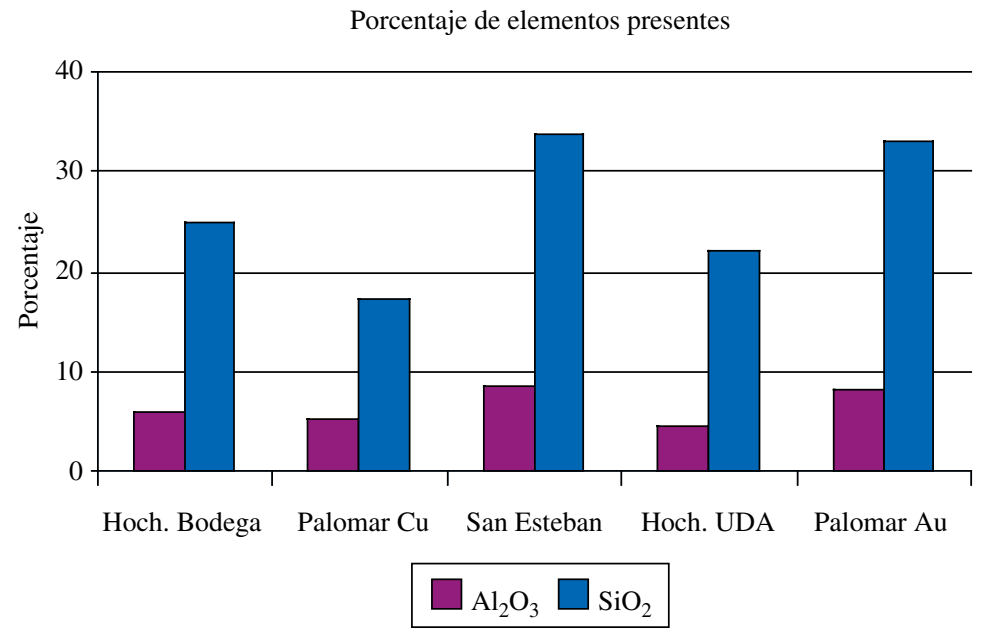

Figura 7. Porcentaje de concentración relaves.

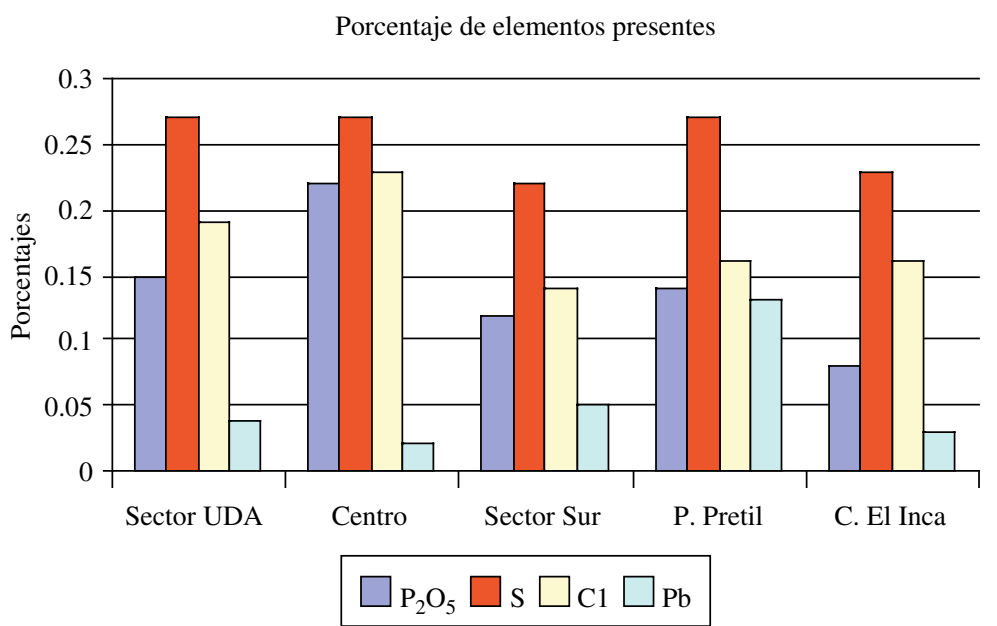

Figura 8. Porcentaje de concentración particulado a nivel de techumbres.

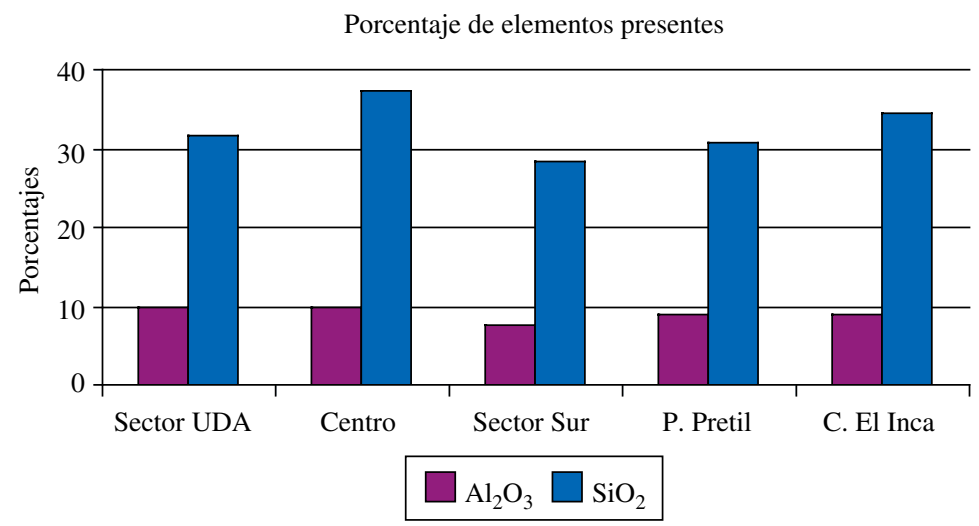

Figura 9. Porcentaje de concentración particulado a nivel de techumbres. 


\section{LITERATURA CONSULTADA}

CELIS, J.E.; MORALES, J.R.; ZAROR, C.A.; CARVACHO, O.F. 2007. Contaminación del Aire Atmosférico por Material Particulado en una Ciudad Intermedia: El Caso de Chillán (Chile).Información Tecnológica. Vol 18 (3), 49-58 (2007).

BRUNEKREE F.B.; FORSBERG, B. 2005. Evidencia epidemiológica de los efectos de las partículas gruesas aerotransportadas en la salud. European Respiratory Journal 6; 309-318 (2005)

VALLEJO, L.R.; FUENTESECA, J.W.; ROMO-KROGER, C.M.; MORALES, J.R.; LLONA, F. 1991. Efecto del traslado y embarque de minerales sobre la calidad del aire en una ciudad puerto de Chile. Interciencia; Julio-Agosto 1991, vol. $16 \mathrm{~N}^{\circ} 4$ (1991).
CONAMA R.M. 1996. Estudio: Caracterización y cuantificación de las distribuciones de material particulado respirable. Conama RM - U. de Sao Paulo - USACH (1996).

ALDAPE, F.; FLORES, J.; DÍAZ, R.; MIRANDA, J.; CAHILL, T.; MORALES, J. 1991. Seasonal Study of the Composition of Atmospheric Aerosols in Mexico City; Int. J. PIXE 1, 355-371 (1991).

SESMA (Servicio de Salud Metropolitano del Ambiente). Caracterización de elementos inorgánicos presentes en el aire de la Región Metropolitana 1997-2000. Ministerio de Salud, Laboratorio de Salud Ambiental, Santiago de Chile, 42 p. (2002). 